\title{
Chaos and thermal conductivity
}

\author{
Silvia Corezzi, ${ }^{1}$ Marco Bianucci, ${ }^{1}$ and Paolo Grigolini ${ }^{1,2,3}$ \\ ${ }^{1}$ Dipartimento di Fisica dell'Università di Pisa, Piazza Torricelli 2, 56100 Pisa, Italy \\ ${ }^{2}$ Istituto di Biofisica del Consiglio Nazionale delle Ricerche, Via San Lorenzo 26, 56127 Pisa, Italy. \\ ${ }^{3}$ Department of Physics of the University of North Texas, P.O. Box 5368, Denton, Texas 76203
}

(Received 5 July 1995)

\begin{abstract}
We argue that the condition of local thermal equilibrium realized several years ago by Rich and Visscher [Phys. Rev. B 11, 2164 (1975)] through a process of mathematical convergence can be obtained dynamically by adopting the prescription of a recent paper [M. Bianucci, R. Mannella, B. J. West, and P. Grigolini, Phys. Rev. E 51, 3002 (1995)]. This should contribute to shedding light on the still unsolved problem of the microscopic derivation of the heat Fourier law.
\end{abstract}

PACS number(s): 05.45. $+\mathrm{b}, 44.10 .+\mathrm{i}, 66.10 . \mathrm{Cb}$

In a recent review paper, Ford [1] has passionately stressed the connection between deterministic chaos and thermal conductivity, reaching the conclusion that "deterministic randomness (chaos) in the sine qua non for a proper thermal conductivity." Yet, in his review paper he strongly emphasizes that a rigorous derivation is still lacking for fundamental reasons related to the difficulty of properly defining chaos in general, and to the distressing conclusion that "distinguishing integrable from nonintegrable systems is, in general, no easier than distinguishing rational from irrational numbers."

We set aside this and other, probably related, questions concerning a genuine approach to irreversibility [2], such as the quantum mechanical nature of the microscopic systems, the conflict between the assumption of a rigorously exponential behavior, and the constraints imposed on relaxation by quantum and classical mechanics [3]. Here we want to limit ourselves to substantiating the main argument of Ford with physical reasoning, rather than by means of rigorous mathematical arguments, these latter being probably unavailable to anybody within the current theoretical paradigms, regardless of his or her mathematical ability and talent. Ford stresses [1] that the ding-a-ling model [4] has the key ingredient for the ambitious task of producing ordinary thermal conductivity, and this ingredient is deterministic chaos. We completely agree with Ford on this key issue. However, we think that even within the limitations stemming from the provisos earlier stressed, the reason chaos results in thermodynamics and statistical mechanics has not yet been clearly assessed. This is probably related to another of the reasons that Ford illustrates to further his belief that, although promising, all the important results obtained so far, including perhaps the ding-a-ling model [4], cannot yet be considered the final solution of the problem of the microscopic derivation of the Fourier law. This is probably so because there is still a large gap to fill between dynamics and statistical mechanics. In other words, the kind of thermodynamics one derives from chaos seems to fit the formal prescriptions of a generalized version of thermodynamics [5], but it is difficult to recover out of it ordinary thermodynamics, the thermodynamics behind the Fourier law, the only one that less sophisticated investigators would recognize as real thermodynamics.
Furthermore, there exists an apparent conflict between the recent research work stressing the role of chaos and nonlinearity and the conventional wisdom according to which linear modeling along the lines of Debye [6] has still to be regarded as being a good departure point for studying condensed systems and the related processes of transport, including thermal transport [6].

We want to show here that a promising road to the ambitious purpose of shedding light on these issues might be realized by making joint use of two distinct theoretical results, the former published several years ago [7] and the latter just appeared [8]. Let us discuss first the proposal of Visscher and co-workers [7]. We shall illustrate this proposal, originally applied to the case of a very large number of atoms, by applying it to the very simplified case of an atomic chain of only four atoms [Fig. 1(a)]: this is a number of atoms large enough to illustrate the essence of the interesting proposal of these authors [7], while keeping the treatment at a merely analytical level. Each atom of the harmonic chain interacts with its own independent thermal bath with the following proviso: the terminal particles of the chain, the first and the fourth particles, interact with two thermal sources whose temperatures $T_{1}$ and $T_{4}$, respectively, are arbitrarily fixed, whereas the baths of the second and third particles are characterized by temperatures that have to be properly adjusted according to the prescription [7] that there is no energy flow between the chain atom and its bath in the steady state.

From a formal point of view, the system is described by the following set of equations:

$$
\begin{aligned}
& \dot{x}_{1}=v_{1}, \\
& \dot{v}_{1}=\omega^{2}\left(x_{2}-x_{1}\right)-\omega^{2} x_{1}-\lambda_{1} v_{1}+f_{1}(t), \\
& \dot{x}_{2}=v_{2}, \\
& \dot{v}_{2}=\omega^{2}\left(x_{3}-x_{2}\right)-\omega^{2}\left(x_{2}-x_{1}\right)-\lambda_{2} v_{2}+f_{2}, \\
& \dot{x}_{3}=v_{3}, \\
& \dot{v}_{3}=\omega^{2}\left(x_{4}-x_{3}\right)-\omega^{2}\left(x_{3}-x_{2}\right)-\lambda_{3} v_{3}+f_{3}, \\
& \dot{x}_{4}=v_{4}, \\
& \dot{v}_{4}=-\omega^{2}\left(x_{4}-x_{3}\right)-\omega^{2} x_{4}-\lambda_{4} v_{4}+f_{4},
\end{aligned}
$$


where the $x_{i}$ 's denote the spatial shifts from the equilibrium positions. The stochastic forces $f_{i}, i=1,4$ (Gaussian white noises), represent fluctuations and the terms $-\lambda_{i} \nu_{i}$, $i=1,4$, denote the corresponding dissipation processes: these are the fluctuation-dissipation processes resulting from the coupling of the particles of the system to their respective baths. For simplicity we assign to the oscillations of the particles about their equilibrium position the common frequency $\omega$. Let us consider first the case where the second and third particles are not coupled to their baths $\left(\lambda_{i}=0, f_{i}=0\right.$, for $\left.i=2,3\right)$. By adopting a formal matrix representation, it is possible to determine the equilibrium distribution of kinetic energy and, consequently, the steady temperature distribution [9]. Using the choice $T_{1}=10.0, T_{2}=10.5, \lambda=1, \omega^{2}=0.5$ (in suitable

(a)

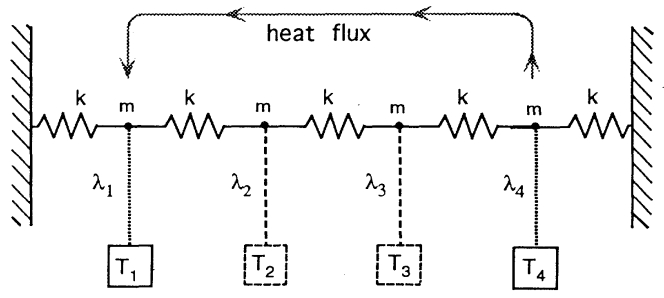

(b)

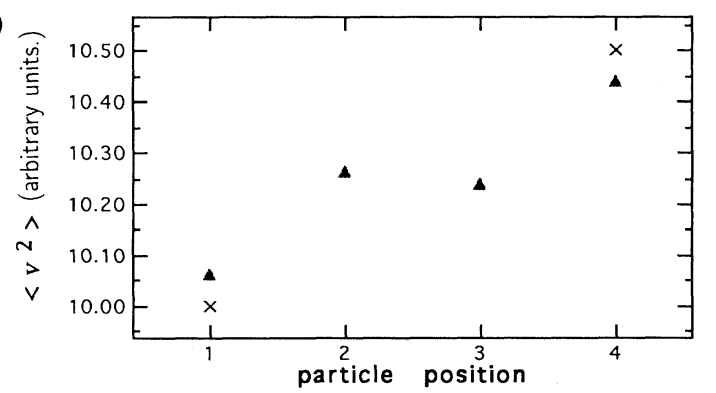

(c)

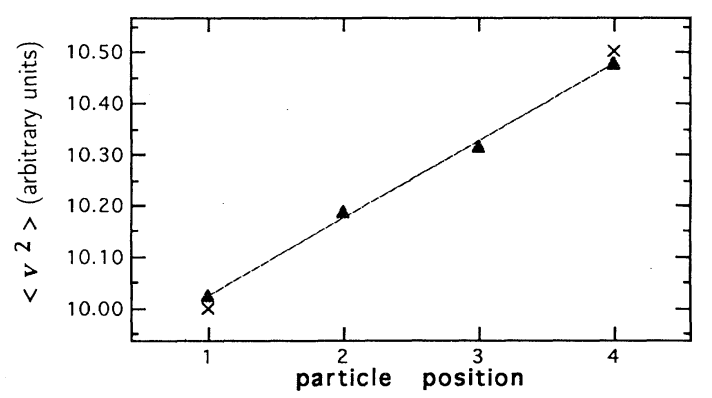

FIG. 1. Model for the harmonic chain with self-consistent baths [7]. (a) Schematic representation. The temperatures of the intermediate baths (boxes with dashed contours) have to be determined by adopting the self-consistent prescription of Ref. [7]. For further details see the text. (b) Kinetic energy distribution (in arbitrary unity) corresponding to the case where the intermediate particles are decoupled from their baths. The crosses denote the fixed temperatures of the end baths and the triangles denote the mean squared values of the particle velocities corresponding to the stationary condition. (c) Kinetic energy distribution (in arbitrary unity) corresponding to the case where the intermediate particles are coupled with their baths and the self-consistent prescription of Rich and Visscher [7] is applied. The meaning of the symbols is the same as in (b). arbitrary units) we obtain $\left\langle v_{1}^{2}\right\rangle=10.0618,\left\langle v_{2}^{2}\right\rangle$ $=10.2618,\left\langle v_{3}^{2}\right\rangle=10.2382$, and $\left\langle v_{4}^{2}\right\rangle=10.4382$, illustrated in Fig. 1(b).

The situation changes drastically if we adopt the prescription of mathematical self-adjustment suggested by Visscher and co-workers [7]. This means also that the second and the third particles are coupled to a bath. However, the temperatures of these intermediate baths are not fixed. Rather they have to be determined by an iterative process of self-adjustment based on the assumption that the stationary state corresponds to a vanishing heat flow between the intermediate particle considered $(i=2,3)$ and its bath. This condition can be implemented in the preceding scheme of calculation and leads to the result $T_{2}=10.185, T_{3}=10.315$ for the intermediate particles [9]. Figure 1(c) illustrates the new temperature distribution. The linear dependence of temperature on the position, and the big transition from the infinite thermal conductivity of the case of Fig. 1(b) to a condition of finite conductivity (when the adoption of a very large number of atoms makes it possible for us to adopt the continuum space approximation), is already evident.

These results, as simple as they are, being derived by means of a fully analytical treatment, have, in our opinion, some appealing aspects. The model of Visscher and co-workers [7] is linear, and this is the reason it can be solved exactly. On the other hand, it refers to that kind of idealization of reality (see the Debye approach) which has been proved to lead to a satisfactory solution of the heat capacity [6]. Nonlinearity and chaos, the basic ingredients stressed by Ford, are tacitly present through the fluctuation-dissipation processes representing the interaction among the intermediate particles and their thermal baths.

Furthermore, the procedure of mathematical convergence prescribed by Visscher and co-workers [7] is exactly equivalent to the physical process of temperature readjustment that intermediate thermal baths with a finite thermal capacity would have. Formally, this key aspect can be easily accounted for as follows. One writes the multidimensional Fokker-Planck equation corresponding to the system of (1) with also the second and the third particles coupled to their baths. However, rather than making the assumption that they have an infinite thermal capacity, thus keeping fixed the temperatures $T_{2}$ and $T_{3}$, we let them change according to the amount of energy exchanged with the particles to which they are coupled.

The amount of heat exchanged is determined by

$$
d Q_{i} / d T_{i}=C_{i}, \quad i=2,3,
$$

where $C_{i}$ denotes the finite thermal capacity of the $i$ th bath. From the multidimensional Fokker-Planck equation we get

$$
d Q_{i} / d t=-\lambda_{i}\left(k_{B} T_{i}-\left\langle v^{2}\right\rangle_{i}\right), \quad i=2,3,
$$

where $\lambda_{i}$ indicates the friction corresponding to the dissipation terms of Eq. (1). By joint use of these equations we obtain

$$
d T_{i} / d t=-\lambda_{i}\left(k_{B} T_{i}-\left\langle v^{2}\right\rangle_{i}\right) / C_{i}, \quad i=2,3 .
$$


We see from Eq. (4) that when the steady state is reached, the condition of a vanishing heat flow between each intermediate particle and its bath is automatically realized, thereby ensuring the equivalence between the process of mathematical convergence of Visscher and co-workers [7] and the process of self-adjustment of the intermediate baths, triggered by their finite heat capacity.

At this stage we have to properly use the results of Ref. [8]. This paper shows that if a particle of interest is coupled to a chaotic system, termed booster to point out its mechanical rather than thermodynamical nature, the dynamical properties of the particle of interest become indistinguishable from that of a Brownian particle, namely, a particle driven by an ordinary Fokker-Planck equation, with a friction $\lambda_{i}$ and a diffusion $D_{i}=\lambda_{i} k_{B} T_{i}$. If the kinetic energy of the particle is given a value not corresponding to the equilibrium condition of the particle, this is expected to change with a rate given by (3). This means that the microscopic derivation of the Fourier law implies that each particle of the intermediate set, namely, $i=2,3$, has to be coupled with its own booster, a mechanical system at a given energy $E_{i}$, with $i=2,3$. In [8] the change of energy affecting the booster was disregarded. However, it is straightforward to correct that flaw in the theory of Ref. [8]: this can be done by using the prescriptions, themselves, of this theory. Let us apply this theoretical derivation. The booster serves the basic role of changing mechanical energy into something that can be perceived as heat, if we look at it from the system of interest. Thus, it is possible to derive a mechanical expression for the temperature $T_{i}$ which is a given function of the energy $E_{i}$. From the expansion

$$
\begin{aligned}
k_{B} T_{i}\left(E_{i}+\Delta E_{i}\right)= & k_{B} T_{i}(E)+k_{B} \Delta E_{i} / C_{i} \\
& +O\left(\Delta E_{i}^{2}\right), \text { with } i=2,3,
\end{aligned}
$$

we derive an expression for the thermal capacity of the intermediate baths, which leads us to an equation equivalent to (4) and, consequently, to a process of temperature adjustment equivalent to the prescription of Visscher and co-workers [7]. Of course, if the booster is characterized by only a few degrees of freedom, this condition has to be realized with caution. For the theory of Ref. [8] to apply, it is necessary that the energy change of the booster does not alter the chaotic condition that determines the booster susceptibility: this means that the condition of local thermodynamics implies a moderate exchange of energy between the particle and its booster.

We now want to comment on some benefits stemming from the approach outlined in this Brief Report.

(i) First of all, we explicitly derive the Fourier law from chaos, rather than merely checking that its occurrence accompanies the onset of chaos. This is so because on the basis of [8] we prove that the chaotic boosters coupled to the intermediate particles realize the condition of local thermodynamics that ensures a condition equivalent to that corresponding to the final stage of the iterative mathematical procedure proposed by Visscher and co- workers [7]: a stationary state with a vanishing heat flow between conducting particle and booster.

(ii) We establish a perspective, within which the Debye theory of noninteracting normal modes and the deterministic chaos of Ford harmonically coexist. This is shown schematically as follows. Following the Debye suggestions [6], we adopt the approximation of considering a crystal as a harmonic lattice. This approximation would be incompatible with deterministic chaos and, consequently, according to Ford [1], with the microscopic derivation of the Fourier law of heat transport. However, we imagine that this perfect crystal interacts with an "environment" in such a way that the perfect crystal, the environment, and the coupling between the two happen to be expressed by a Hamiltonian representation identical to the Hamiltonian of the nonideal crystal that we are studying. This nonideal crystal, in turn, is supposed to be a medium suitable for heat transport. Let us make the assumption that the environment, a nonlinear and chaotic system, can be divided into infinitely many subsystems, a subsystem for each atom of the perfect lattice, and that two distinct subsystems do not interact with one another, but only with the atom to which they are associated. The approximation of neglecting the direct interaction among distinct subsystems becomes reasonable with increasing degrees of freedom of these subsystems. As a result of this approximation scheme, we derive a model equivalent to that earlier used to derive the Fourier law. When the stationary condition, corresponding to the Fourier law is reached, the harmonic lattice can be regarded as noninteracting with the network of boosters. The two systems do not exchange energy and, consequently, they can be regarded as two independent channels for the heat transmission. Furthermore, the network of boosters is a system to which, too, the Fourier law applies. This is consistent with the results found by Casati et al. [4], according to whom the Fourier law implies a condition of global chaos. Let us see why: After getting the desired result by coupling each atom to its booster and keeping the boosters isolated from one another, let us switch on again the interaction among distinct boosters. If we consider the collection of all the boosters as a single chaotic system, we expect it to realize the Fourier law. This implies an energy distribution indistinguishable from that obtained before switching on the interaction among distinct boosters. Consequently, the assumption made of noninteracting boosters seems to be reasonable and its release should not lead to a significantly different result. It must be pointed out that although chaos is the key ingredient in deriving the Fourier law, the thermal conductivity associated with a strongly chaotic system is low. Consequently, the contribution to conductivity of the chain of boosters is low, and the errors due to the approximation made (of considering the boosters as noninteracting) should be negligible. In conclusion, our result is compatible with that of Casati et al. [4] and shows how to use the key ingredient of deterministic chaos without losing the benefits stemming from the Debye harmonic model. 
[1] J. Ford, Phys. Rep. 213, 271 (1992).

[2] P. Grigolini, Quantum Irreversibility and Measurement (World Scientific, Singapore, 1993).

[3] M. H. Lee, Phys. Rev. Lett. 51, 1227 (1983).

[4] G. Casati, J. Ford, F. Vivaldi, and M. W. Visscher, Phys. Rev. Lett. 52, 1861 (1984).

[5] D. Ruelle, Thermodynamic Formalism, the Mathematical Structures of Classical Equilibrium Statistical Mechanics, Encyclopedia of Mathematics and its Applications, Vol. 5
(Addison-Wesley, Reading, MA, 1978).

[6] C. Kittel, Thermal Physics (Wiley, New York, 1969).

[7] M. Rich and W. M. Visscher, Phys. Rev. B 11, 2164 (1975); M. Bolsterli, M. Rich, and W. M. Visscher, Phys. Rev. A 1, 1086 (1970).

[8] M. Bianucci, R. Mannella, B. J. West, and P. Grigolini, Phys. Rev. E 51, 3002 (1995).

[9] S. Corezzi, Diploma thesis, University of Pisa, 1994 (unpublished). 